\title{
Numerical Analysis of Attenuation of Communication Signal in Plasma Sheath
}

\author{
ZHAO Jie, LI Zi cheng \\ ${ }^{1}$ The Engineering and Technical College of Chengdu University of Technology, Leshan 614007, China \\ ${ }^{2}$ Southwestern Institute of Physics, Chengdu 610041, China \\ WANG Shi qing, LIU Jian, XU Li \\ The Engineering and Technical College of Chengdu University of Technology, Leshan 614007, China
}

\begin{abstract}
In order to simulate the communication signals attenuation, the model of plasma sheath is based on the layer theory and the modified double exponential model in blackout. The results of simulation show that: The attenuation constant reaches a peak value when the angular frequency of communication signal is equal to the natural angular frequency of the plasma, and the different collision angular frequency of plasma has different the peak value of attenuation. In addition, the peak value and bandwidth of attenuation is a contradiction. As electron density increases, the attenuation constant increases rapidly. When the temperature is below $1000 \mathrm{~K}$, the attenuation constant increases rapidly, and then reduces slowly.
\end{abstract}

KEYWORD: plasma; electromagnetic wave; attenuation constant; blackout

\section{INTRODUCTION}

When the aircraft passes through the atmosphere with hypersonic, the friction is produced between the surface of vehicle and the air, so strong ionization and non-uniform plasma sheath is generated in the aircraft around. This plasma sheath may interfere with aircraft communications signals, or even cause communication interruption, which is called "blackout" phenomenon [1-4]. For example, when the Gemini spacecraft return to the ground, there have been five minutes communication interruption; when Shen Zhou VII spacecraft passes through the "blackout" area, there have been about four minutes of communications interruption. In addition, it is to improve the accuracy of the missile hitting the target, or satellites and spacecraft's return capsule landing safely, the good communication. Systems are indispensable. In particular, when the manned spacecraft enters the atmosphere, good communication system is an important guarantee for the astronaut life[5].

As early as the 1960 s, research work about the "blackout" phenomenon has been carried out in foreign countries. For example, the United States has invested a lot of money in a communication signal attenuation measurement, getting a lot of technical data and experience[6-7], and in the 1980s, China began to carry out a lot of experimental work. A lot of research work of signal attenuation simulation in the plasma sheath has been done. For example, Laroussi calculated the absorption and reflection power of the electromagnetic waves in twodimensional, magnetized, steady-state, non-uniform plasma, and got the relations of absorbing power and reflected power with plasma parameters[8]; Evans calculated signal attenuation in simulated plasma sheath which is generated by the Chemical combustion experiment[9].HC Kim provided the numerical solution of reflection and transmission coefficient of the electromagnetic waves in a non-uniform plasma layer, and the numerical solution and the theoretical solution are consistent[10]. In this paper, the communication signal attenuation is calculated, and the model of plasma sheath is based on the layer theory and the modified double exponential model in blackout. The effect of the various parameters of the plasma (such as the angular frequency of communication signal, the collision angular frequency of plasma, the electron density, temperature) on communication signal attenuation is discussed. This provides a good way to solve the communication interruption problems in "blackout" area.

\section{THE SIMULATION MODEL}

\subsection{The plasma model in blackout zone}

The plasma is aeolotropy and complex medium[11], and actually the plasma density tends to be zero on the surface of aircraft and far from the surface. At one or several limited distance it has extreme, but the variation of plasma density is continuous in the 
plasma. The direction of $\mathrm{Z}$ axis is in conformity with the normal direction of the aircraft. The distribution of plasma electron density is given by the formula (1) and figure 1.
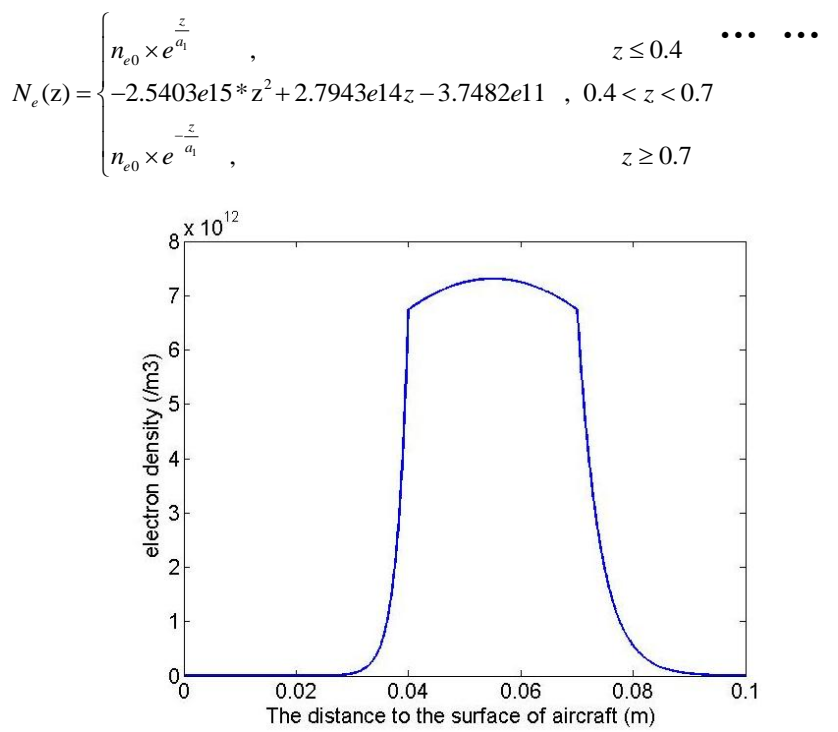

Fig.1 Distribution of electron density in the plasma sheath

\subsection{The relationship between the attenuation and various parameters}

When the electromagnetic wave propagates in the uniform plasma where there are a large number of electron collisions, attenuation constant can be obtained by the Maxwell equations of the electromagnetic wave:

$$
\alpha=\frac{\omega}{C}\left\{\frac{1}{2}\left[-\left(1-\frac{\omega_{p}^{2}}{\omega^{2}+v^{2}}\right)+\left(\left(1-\frac{\omega_{p}^{2}}{\omega^{2}+v^{2}}\right)^{2}+\left(\frac{v}{\omega} \frac{\omega_{p}^{2}}{\omega^{2}+v^{2}}\right)^{2}\right)^{1 / 2}\right]\right\}^{1 / 2} \cdots
$$

Where $\omega=2 \pi f$ is the angular frequency of communication signal (that is the angular frequency of electromagnetic wave), $f$ is the frequency of communication signal and $\omega_{p}$ is the angular frequency of plasma which has a relationship with the electron density distribution.

$$
\omega_{p}=\left(N_{e} e^{2} / m_{e} \varepsilon_{0}\right)^{1 / 2}
$$

Where $m_{e}$ is the mass of electron, $N_{e}$ is the distribution of electron density and $v$ is the collision angular frequency of plasma. Since the number of neutral particles is much larger than the plasma ions in blackout, so the collide of electrons and neutral particles are only considered. The collision angular frequency of plasma $v$ can be written as:

$$
v=2 \pi v_{e n}
$$

Where $v_{e n}=\left\langle v_{e}\right\rangle / \lambda_{e n}$ is the average number of collisions of the electrons and ions and neutral particles in unit time, $\lambda_{e n}=\left(n_{n} \sigma_{e n}\right)^{-1}$ is the mean free path of the electrons and neutral particles, and $n_{n}$ is the density of neutral particles which meets the state equation of ideal gas $p=n_{n} k T$, and the $v_{e}$ is average thermal velocity which can be written as:

$$
\left\langle v_{e n}\right\rangle=\left(\frac{8 k T}{\pi m_{e}}\right)^{1 / 2}
$$

in which $T$ is the temperature, $p$ is the pressure, $\sigma_{e n}=\pi r^{2}$ is collision cross section, $r$ is the radius of the gas molecules.

For the convenience of analysis and calculation, the plasma is divided into $\mathrm{N}$ layers(As shown in Figure 2), supposing the thickness of the simulated plasma is 0.1 meters, and the distribution of electron density is nonlinear. The electronic density can be regarded as the approximate uniform in each layer, then the attenuation is solved in the each layer, next the attenuation of all layers are integrated on the $\mathrm{Z}$ axis, and finally the total attenuation is calculated. So the total attenuation can be written as:

$$
A=8.86 \int_{z 2}^{z 1} \alpha(\mathrm{z}) d z
$$

\begin{tabular}{ll} 
Layer 0 & $\alpha_{1}$ \\
\hline Layer 1 & $\alpha_{2}$ \\
\hline Layer 2 & $\alpha_{3}$ \\
\hline & \\
Layer n-1 & $\alpha_{n-1}$ \\
\hline Layer n & $\alpha_{n}$ \\
\hline
\end{tabular}

Fig.2 Schematic diagram of the layered plasma sheath

\section{THE CALCULATION RESULTS}

\subsection{Effect of the angular frequency of communication signal on attenuation}

Supposing the collision angular frequency of the plasma $v=1.2 \times 10^{10}$, and the angular frequency of communication signal $\omega=10^{7}--10^{12} \mathrm{rad} / \mathrm{s}$, the angular frequency of the plasma are: $\omega_{p}=1.2 \times 10^{11} \mathrm{rad} / \mathrm{s} \quad 、 \omega_{p}=3.9 \times 10^{10} \mathrm{rad} / \mathrm{s}$ 、 $\omega_{p}=5.5 \times 10^{9} \mathrm{rad} / \mathrm{s}$. From the results of Figure 3, when the angular frequency of communication signal is equal to the angular frequency of the plasma, the attenuation of communication signals is the maximum. In other cases the attenuation are relatively small. In addition, the attenuation of communication signal increases with the angular frequency of the plasma, according to the formula (3), which is the attenuation of communication signals increase with the increase of the electron density in the plasma. This result is 
consistent with the interaction theory of electromagnetic waves and plasma.

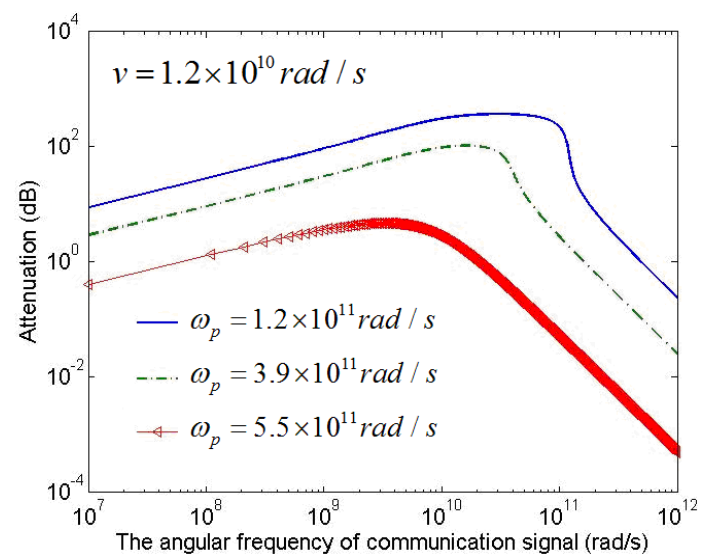

Fig. 3 The relation of attenuation and the angular frequency of communication signal

\subsection{Effect of plasma collision angular frequency on attenuation}

If the angular frequency of the plasma $\omega_{p}=5.5 \times 10^{9} \mathrm{rad} / \mathrm{s}$, when the angular frequency of communication signals $\omega=12 \times 10^{9} \mathrm{rad} / \mathrm{s}$, $\omega=24 \times 10^{9} \mathrm{rad} / \mathrm{s}, \omega=12 \times 10^{10} \mathrm{rad} / \mathrm{s}$, the attenuation of communication signals is calculated with the collision angular frequency of plasma. The results can be seen from Figure 4, when the collision angular frequency of plasma increases, the attenuation of communication signals increases first and then decreases, the peak value and the location of the attenuation of communication is not the same. This phenomenon is due to the plasma collisional absorption, this calculation results is consistent with reference [12]. Figure 3 also shows, the collision angular frequency of plasma increases when the attenuation peak value reduces. But from Figure 4, if the collision angular frequency of plasma increases, the width of the attenuation band is also correspondingly increased. So there is a contradiction between the attenuation band and the attenuation peak, and they can not be satisfied simultaneously.

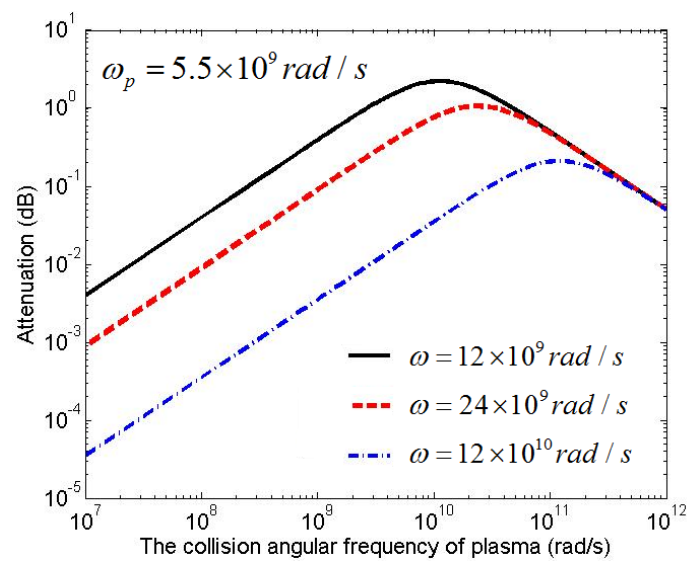

Fig. 4 The relation of attenuation and the collision angular frequency of plasma

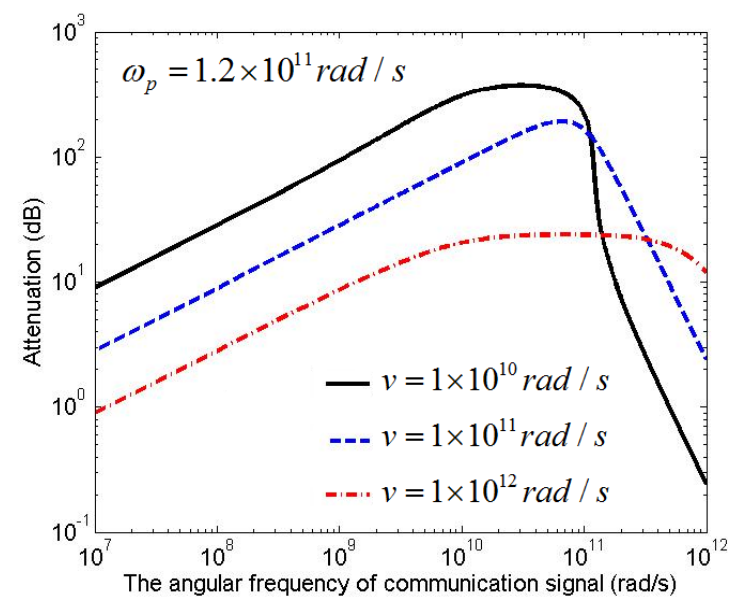

Fig.5 The relation of attenuation and the angular frequency of communication signal

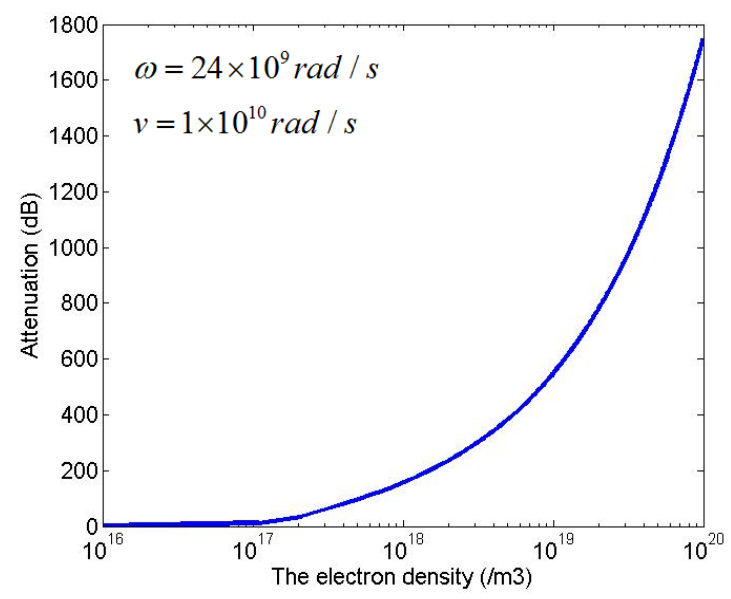

Fig.6. The relation of attenuation and electron density

\subsection{Effect of Electron density on attenuation}

The C-band is used in communication of aircraft, so selecting the angular frequency of the communication signal $\omega=2.4 \times 10^{10} \mathrm{rad} / \mathrm{s}$, the collision angular frequency of plasma is $v=1 \times 10^{10} \mathrm{rad} / \mathrm{s}$. Effect of Electron density on attenuation is calculated. Figure 6 shows that: the attenuation is small when the electron density is less than $10^{18} / \mathrm{m}^{3}$, and it changes little; when the electron density is greater than $5 \times 10^{19} / \mathrm{m}^{3}$, the attenuation is greater than $1000 \mathrm{db}$, then it quickly increases. Therefore, reducing the electron density of plasma can help reduce effect of plasma on attenuation communication signal. This provides a good way to solve the communication interruption problems in "blackout" area.

\subsection{Effect of temperature on attenuation}

Supposing the angular frequency of the plasma is $\omega_{p}=5.5 \times 10^{9} \mathrm{rad} / \mathrm{s}$, when the angular frequency of communication signals is $\omega=2.4 \times 10^{10} \mathrm{rad} / \mathrm{s}$, the relationship of the communication signal attenuation and temperature is studied. By equation (4) and (5), 
the relationship can be obtained between the collision angular frequency of plasma and temperature, Figure 7 shows that the collision angular frequency of plasma is inversely proportional to the temperature. Figure 4 shows the relationship of the collision angular frequency of plasma and the attenuation has a peak value, so Figure 8 shows the relationship of the temperature and attenuation also has a peak value. When the temperature is below $1000 \mathrm{~K}$, the attenuation increases rapidly, and then reduces slowly.

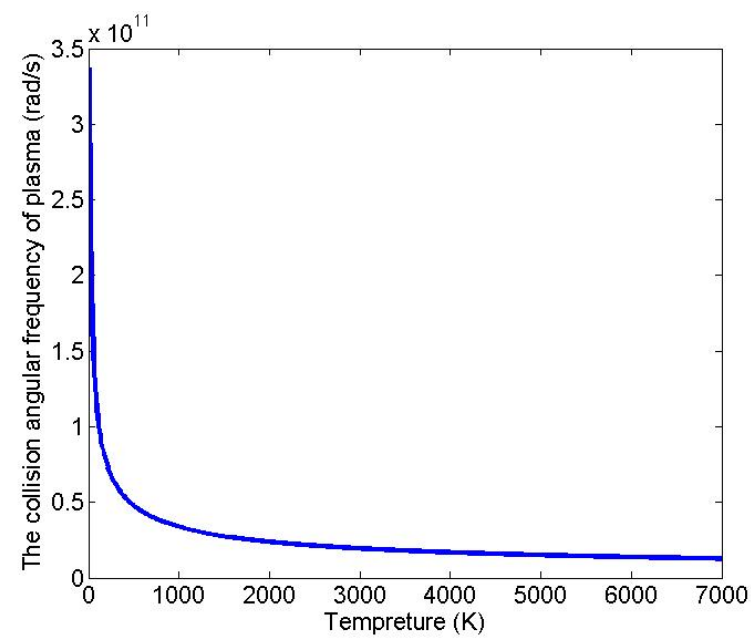

Fig.7 The relation of temperature and plasma collision angular frequency

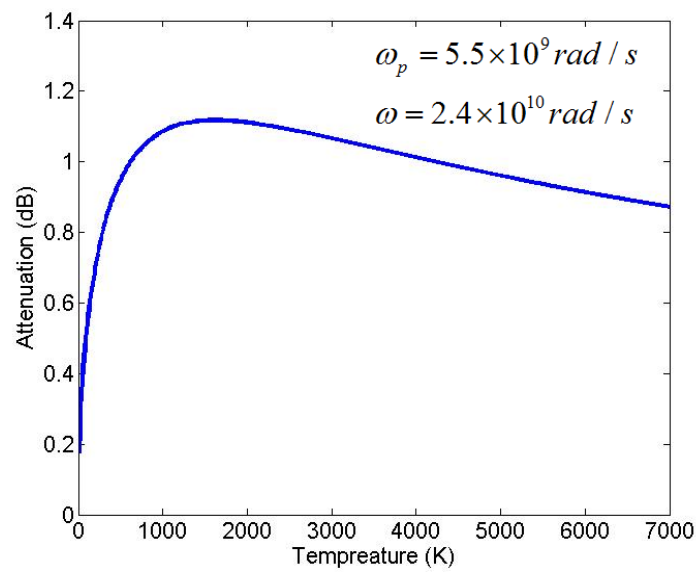

Fig. 8 The relation of temperature and attenuation

\section{CONCLUSIONS}

The model of plasma sheath is established based on the layer theory and the modified double exponential model in blackout. In addition, the attenuation is obtained by Maxwell's equations. The following results are obtained by calculation:

(1)As the frequency of communication signal increases, the attenuation increases first, and then decreases. When the angular frequency of communication signals is equal to the angular frequency of the plasma, the attenuation reaches a peak value and in other cases the attenuation are relatively small.

(2)The different collision angular frequency of plasma has different the peak value of attenuation. In addition, the peak value and bandwidth of attenuation is a contradiction.

(3) The attenuation is small when the electron density is less than $10^{18} / \mathrm{m}^{3}$, and it changes little. When the electron density is greater than $5 \times 10^{19} / \mathrm{m}^{3}$, the attenuation constant is greater than $1000 \mathrm{db}$, and then it quickly increases.

(4) When the temperature is below $1000 \mathrm{~K}$, the attenuation constant increases rapidly, and then reduces slowly.

\section{ACKNOWLEDGEMENTS}

National Natural Science Foundation of China (No.11375055)

College Science Research and Development Fund (No. C122009015)

\section{REFERENCES}

[1] RybakJ P, Churchill RJ. 1971, IEEE Transactions on Aerospace and Electronic Systems, 7(5): 879-894.

[2] Wang Bai yi. 1982, Journal of Astronautics, (2):81-101. (in Chinese)

[3] Hartunian R A, Stewart GE, RavnO. Jan. 2007, Cause sand mitigation of radio frequency blackout during reentry of reusable launch vehicles. El Segundo: Aerosp. Corporation, ATR-2007 (5309-1)

[4] Potter DL, 2006, Introduction of the PIRATE program for parametric reentry vehicle plasma effects studies. The 37 th AIAA Plasma dynamics and Lasers Conference, San Francisco, USA.

[5] C. R. Mullin, F. H. Mitchell. 1968, Proceedings of the IEEE, 56(2):203 204.

[6] Evans J S, and Huber P W. 1963-12, NASN TN, D-2403

[7] Evans J S, Huber P W, Schexnayder C J Jr .1971,. AIAA Journal, 9(6): 1154-1162

[8] M. Laroussi. J. R. Roth. 1993, IEEE Transaction on Plasma Science, 21(3):366 372.

[9] John S. Evans and Paul W.Huber. 1963, National Aeronautics and Space Administration. December Technical Note D-2403.

[10] H. C. Kim, J. P. Verboncoeur. 2007, Comput. Phys. Commun, 177: 118-121

[11] Chai Lin. 2008, Acta Aeronautica ET Astronautica Sinica, 29(4): 1007-1012. (in Chinese)

[12] Liu Shao bin, Mo Jin jun, Yuan Nai chang. 2003, Chinese Journal of Electronics, 31(3):372 375. (in Chinese) 